\title{
DISTRIBUTION OF ENERGY DENSITY OF DIRAC FIELD IN THE CLOSED UNIVERSE
}

\author{
S.K. Sharma* and U. Khanal* \\ *Central Department of Physics, Tribhuvan University, Kirtipur, Nepal.
}

\begin{abstract}
The behavior of the energy of the free Dirac field in the closed FRW universe has been looked at. It is generally expected that the comoving energy density for the particles is conserved. Although this expectation is true for the massless fields, it is not for the massive ones. So, there is a finite energy current in any finite sized volume of the universe. The energy current is positive throughout for lowest state, $n=0$. For $n=1$, however, there occurs negative energy flow near $r=\pi / 2$. So energy flows out of the region. It may be expected that the two regions, on either sides may become disjoint over time, but this can be confirmed only after studying the time evolution. If confirmed, this could explain the process of fragmentation of large structures. Larger n appears to produce larger number of such density contrasts.
\end{abstract}

Key words: Closed Universe; Dirac field; Energy Density.

\section{INTRODUCTION}

In the earlier work [2], the Newman-Penrose formalism [1,2,3] was used to separate the variables and solve the free Dirac field equation in the Friedmann-Roberston-Walker (FRW) spacetime. The FRW metric can be written as

$d s^{2}=a^{2}(\eta)\left[d \eta^{2}-d r_{*}^{2}+\frac{\sin ^{2} \sqrt{K} r_{*}}{K}\left(d \theta^{2}+\sin ^{2} \theta d \phi^{2}\right)\right]$.

Here, a is the scale factor, $\eta=\int \frac{d t}{a}$ is the conformal time and $r_{*}$ is the tortoise radial coordinate that is related to the usual radial coordinate by $r_{*}=\int d r / f=\frac{2}{\sqrt{K}} \arctan \frac{\sqrt{K} r}{2}$, and $K=(0,+1$ or -1$)$ for the flat, closed or open universe respectively. In the work, among other things, the spatial part of the Dirac equation was completely solved. In this paper, we will concentrate on the solution for the closed universe case and discuss the distribution of energy in the Dirac field.

\section{DIRAC EQUATION}

It was shown [2] that the equations governing the free Dirac field reduce to

$\frac{1}{R_{\mp}}\left(\frac{\partial}{\partial r_{*}} \mp \lambda \frac{f}{r}\right) R_{ \pm}=\frac{1}{T_{ \pm}}\left(\frac{\partial}{\partial \eta} \pm i M a\right) T_{\mp}=i k$ where $\mathrm{k}=$ pa is the comoving momentum, $l$ in $\lambda=(l+1 / 2)$ is the total angular momentum including the spin as well and hence is a half-integer. The coupled equation (2) can be written as two pairs of decoupled radial and temporal equations for each of different cases flat, closed and open universes. The radial solutions that are regular at the origin, for the closed universe $(K=1)$, are

$R_{ \pm}=A_{ \pm} \sin ^{l+1 \mp \frac{1}{2}}\left(\frac{n_{n}}{2}\right) \cos ^{l+1 \pm \frac{1}{2}}\left(\frac{r_{k}}{2}\right) P_{k-l-1}^{\left(l+\frac{1}{2} \mp \frac{1}{2}, l+\frac{1}{2} \pm \frac{1}{2}\right)}\left(\cos r_{*}\right) \ldots \ldots$

where $P_{n}^{(\alpha, \beta)}(x)$ is Jacobi Polynomial [4]. This solution for the closed universe is regular at $r_{*}=\pi$, the maximum value that $r_{*}$ can have, only if $\mathrm{k}-1-1$. Although such a quantization of momentum has appeared in the context of closed FRW model, this may be a general rule for all gravitationally bound massive particles.

Writing $x=\cos r_{*}, \alpha=l+\frac{1}{2} \mp \frac{1}{2}, \beta=l+\frac{1}{2} \pm \frac{1}{2}$ and $\mathrm{n}=\mathrm{k}-\mathrm{l}$ -1 , equation (3) can be written as

$R_{ \pm}=A_{ \pm} 2^{-\frac{1}{2}(\alpha+\beta+1)}(1-x)^{\alpha / 2+1 / 4}(1+x)^{\beta / 2+1 / 4} P_{n}^{(\alpha, \beta)}(x)$

Using the normalization condition: $\int_{0}^{\pi}\left|R_{ \pm}\right|^{2} d r_{*}=1$, i.e.,

$\left|A_{ \pm}\right|^{2} 2^{-(\alpha+\beta+1)} \int_{-1}^{1}(1-x)^{\alpha}(1+x)^{\beta}\left\{P_{n}^{(\alpha, \beta)}(x)\right\}^{2} d x=1, \ldots$.

we get $\left|A_{ \pm}\right|^{2}=\frac{\Gamma(n+1) \Gamma(\alpha+\beta+n+1)(\alpha+\beta+2 n+1)}{\Gamma(\alpha+n+1) \Gamma(\beta+n+1)}$,

Author for Correspondence: U. Khanal, Central Department of Physics, Tribhuvan University, Kirtipur, Nepal. Email: khanalu@yahoo.com. 
or, $\left|A_{ \pm}\right|^{2}=\frac{\Gamma(n+1) \Gamma(2 l+n+2)(2 l+2 n+2)}{\Gamma(l+n+1) \Gamma(l+n+2)}$

\section{ENERGY}

The energy momentum tensor quoted in reference [2], let's consider the energy density given by its $\eta-\eta$ component:

$a^{4} T_{\eta}^{\eta}=\rho a^{4}=\frac{i}{8 \operatorname{Sin}^{2} r_{-}} \mid Z_{+}^{*} \frac{\partial Z_{+}}{\partial \eta}+Z_{-}^{*} \frac{Z_{-}}{\partial \eta}-$ complex conjugate $\mid\left[\left.Y_{+}\right|^{2}+\left|Y_{-}\right|^{2}\right] \ldots$

where $\mathrm{Z}=\mathrm{RT}$ represents the radial and temporal wavefunction, and $\mathrm{Y}$ the angular. Integrating over a solid angle, using the normalization condition $\int\left|Y_{ \pm}\right|^{2} d \Omega=1$, we get

$4 \pi \rho a^{4}=\frac{1}{4 \operatorname{Sin}^{2} r_{-}}\left[Z_{+}^{*} \frac{\partial}{\partial \eta} Z_{+}+Z_{-}^{*} \frac{\partial}{\partial \eta} Z_{-}-c c\right]$

The energy flow rate, after using (2) and simplifying, is given by

$\partial_{n}\left(\rho a^{4}\right)=\frac{-M \dot{a}}{4\left(4 \pi \operatorname{Sin}^{2} r_{*}\right)}\left[\left|Z_{+}\right|^{2}-\left|Z_{-}\right|^{2}\right]$

Integrating this over the three-space volume and simplifying, we find the total energy [5] flowing out of the volume of radius $r_{*}$ to be

$\partial_{n}\left(E a^{4}\right)=\frac{M \dot{a}}{2 i k}\left(T_{+}^{2}-1\right) R_{+}^{*} R_{-} \frac{M \dot{a}}{2}\left(1-2\left|T_{+}\right|^{2}\right) \int_{0}^{n}\left|R_{+}\right|^{2} d r_{*}$

At $\eta \rightarrow 0,\left|T_{ \pm}\right|^{2} \rightarrow \frac{1}{2}, \partial_{n}\left(E a^{4}\right)=\frac{M a}{2 i k}\left(\left|T_{+}\right|^{2}-1\right) R_{+}^{*} R_{-}$

If we take the integration limit for $\mathrm{r}$ as $(0, \pi)$, obviously $\mathrm{R}_{ \pm}(0)$ $=\mathrm{R}_{ \pm}(\pi)=0$. So the total comoving energy $\mathrm{Ea}^{4}$ remains constant. At any $\mathrm{r}<\pi$, however, there does occur flow of energy from one region to another. For the closed universe ( $K=1$ ), using the value of $R_{ \pm}$given by equation (4) along with the normalized $\mathrm{A}_{ \pm}$given by equation (6) noting the fact that $\mathrm{A}_{-}=i \mathrm{~A}_{+}$, we have

$\frac{\partial}{\partial \eta}\left(E a^{4}\right)=-\frac{M \dot{a}}{4 k}\left|A_{ \pm}\right|^{2} 2^{-2 l-2}\left[\left(1-\operatorname{Cos} r_{*}\right)^{l+1 / 2}\left(1+\operatorname{Cos} r_{*}\right)^{l+1 / 2}\right]$

$\left\{P_{n}^{(l, l+1)}\left(\operatorname{Cos} r_{*}\right) P_{n}^{(l+1, l)}\left(\operatorname{Cos} r_{*}\right)\right\}$

Since $\frac{d}{d \eta}=\dot{a} \frac{d}{d a}$, the rate of flow of energy per unit mass is given by

$\frac{1}{M} \frac{\partial}{\partial a}\left(E a^{4}\right)=-\frac{1}{4 k}\left|A_{ \pm}\right|^{2} 2^{-2 l-2}\left[\left(1-\operatorname{Cos} r_{*}\right)^{l+1 / 2}\left(1+\operatorname{Cos} r_{*}\right)^{l+1 / 2}\right]$

$\left\{P_{n}^{(l, l+1)}\left(\operatorname{Cos} r_{*}\right) P_{n}^{(l+1, l)}\left(\operatorname{Cos} r_{*}\right)\right\}$

Its plots against $r_{*}$ for $l=1 / 2,3 / 2$ and $5 / 2$ for each of $\mathrm{n}=0,1,2$ and 3 have been shown in figures $1 \mathrm{a}, 1 \mathrm{~b}, 1 \mathrm{c}$ and $1 \mathrm{~d}$ respectively.

From the equation (8) with the help of equation (2), the energy density can be written as
$4 \pi \operatorname{Sin}^{2} r_{*} \quad \rho a^{4}\left(r_{*}\right)=\frac{-k}{4}\left\{\left|R_{+}\right|^{2}\left(T_{+}^{*} T_{-}+T_{+} T_{-}^{*}\right)+\left|R_{-}\right|^{2}\left(T_{-}^{*} T_{+}+T_{-} T_{+}^{*}\right)\right\}$

$+\frac{M a}{2}\left[Z_{-}^{2}-Z_{+}^{2}\right]$

At $\eta \rightarrow 0$, i.e., a $\rightarrow 0,\left|T_{ \pm}\right|^{2} \rightarrow \frac{1}{2}$, this can simply be written as

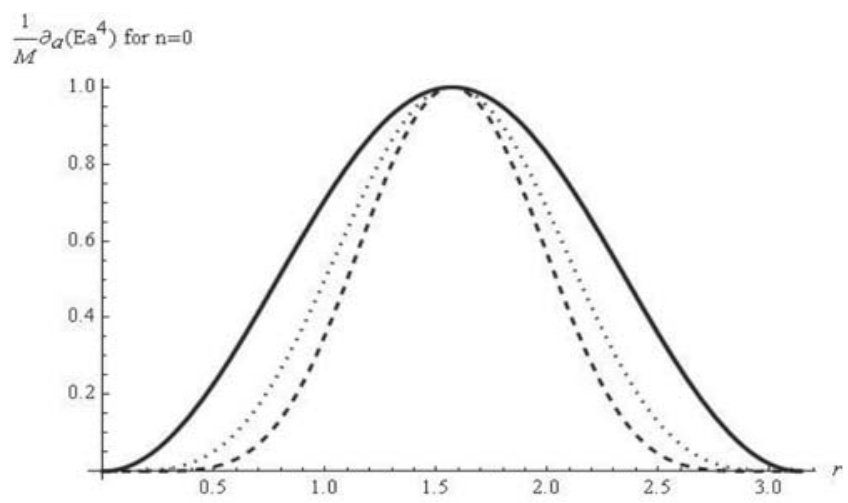

Fig. 1a: The rate of flow of comoving energy per unit mass for $\mathrm{n}=0$. The solid, dotted and dashed curves are for $l=1 / 2,3 / 2$ and $5 / 2$ respectively. The maximum energy increase is near $r=\pi / 2$. Larger $l$ makes the peak sharper.

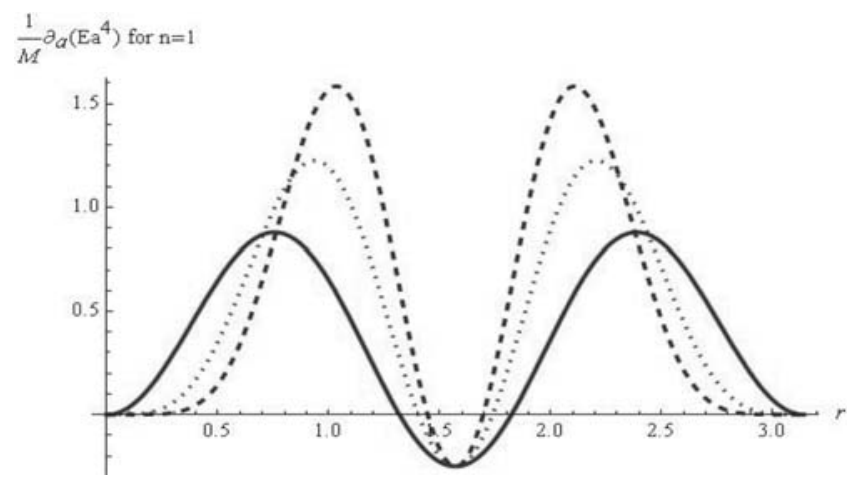

Fig. 1b: The rate of flow of comoving energy per unit mass for $\mathrm{n}=1$. The solid, dotted and dashed curves are for $l=1 / 2,3 / 2$ and $5 / 2$ respectively. There occurs negative energy flow from near $r=\pi / 2$. So energy flow out the region. It may be expected that the two regions, on either sides may become disjoint over time, indicating the process of fragmentation large structures.

$\frac{1}{M} \partial_{a}\left(\mathrm{Ea}^{4}\right)$ for $\mathrm{n}=2$

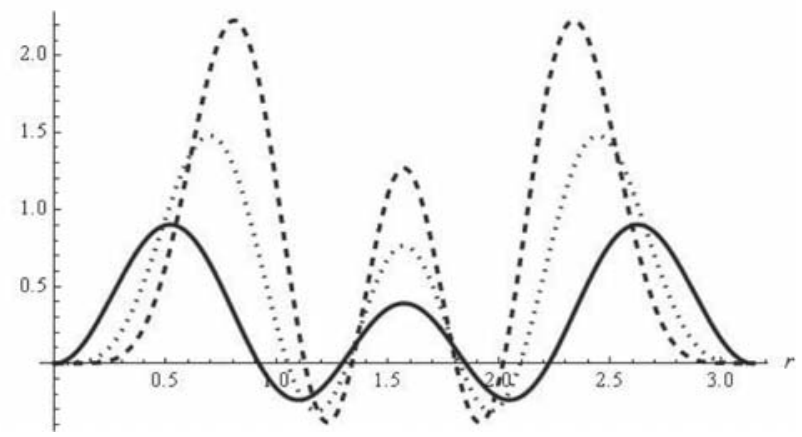

Fig. 1c: As in 1c for $n=2$, its showing more number of density contrasts. 


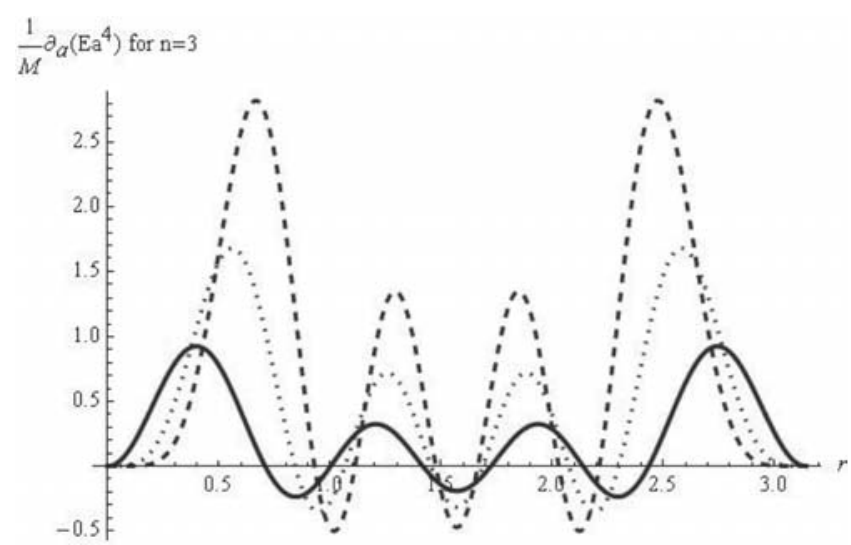

Fig. 1d: As in 1c for $n=3$, its showing more number of density contrasts.

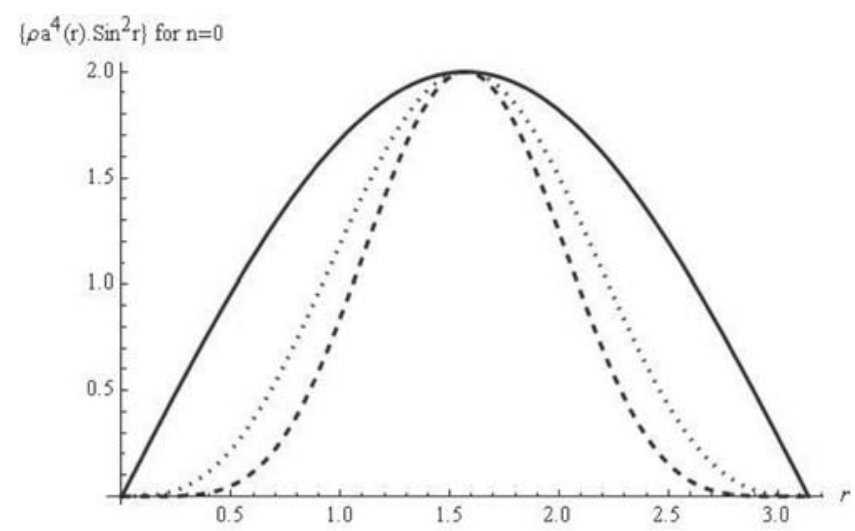

Fig. 2a: The comoving energy density at a distance $r$ for $n=0$. The solid, dotted and dashed curves are for $l=1 / 2,3 / 2$ and $5 / 2$ respectively. The maximum energy density is near $r=\pi / 2$. Larger $l$ makes the peak sharper.

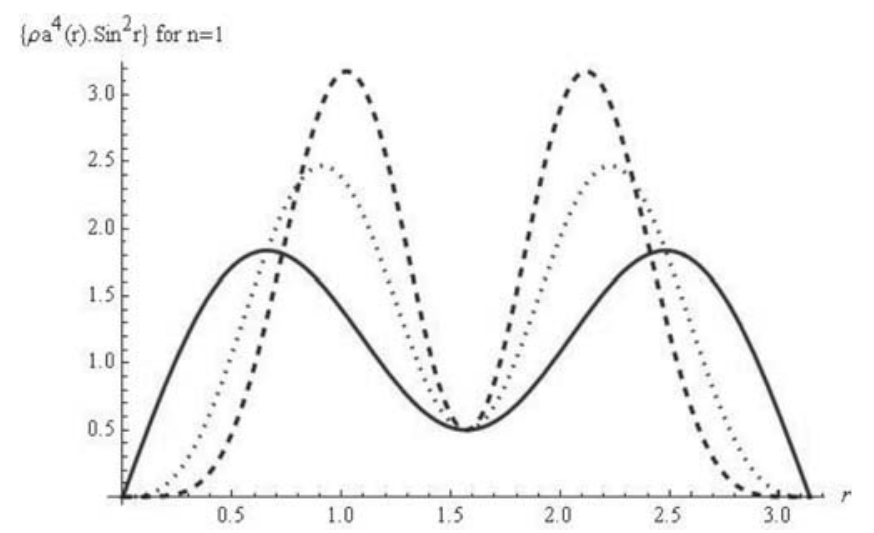

Fig. 2b: The comoving energy density at a distance $r$ for $n=1$. The solid, dotted and dashed curves are for $l=1 / 2,3 / 2$ and 5/2 respectively. It may be expected that the two regions, on either sides may become disjoint over time, indicating the process of fragmentation large structures.

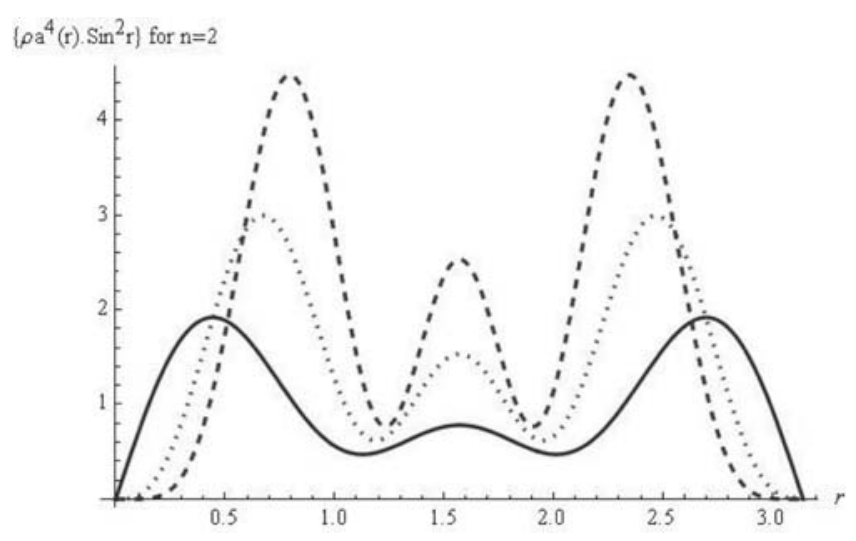

Fig. 2c: As in $2 b$ for $n=2$.

$4 \pi \operatorname{Sin}^{2} r_{*} \rho a^{4}\left(r_{*}\right)=\frac{k}{4}\left\{\left|R_{+}\right|^{2}+\left|R_{-}\right|^{2}\right\}$

$=\left|A_{ \pm}\right|^{2} 2^{-2 l-6}\left[\left(1-\operatorname{Cos} r_{*}\right)^{l}\left(1+\operatorname{Cos} r_{*}\right)^{l+1}\left\{P_{n}^{(l, l+1)}\left(\operatorname{Cos} r_{*}\right)\right\}^{2}\right.$

$\left.+\left(1-\operatorname{Cos} r_{*}\right)^{l+1}\left(1+\operatorname{Cos} r_{*}\right)^{l}\left\{P_{n}^{(l+1, l)}\left(\operatorname{Cos} r_{*}\right)\right\}^{2}\right] \ldots$

Its plots against $r$ for $l=1 / 2,3 / 2$ and $5 / 2$ for each of $\mathrm{n}=0,1$ and 2 have been shown in figures 2a, 2b and 2c respectively.

\section{CONCLUSION}

In this paper, we have looked at the behavior of the energy of the free Dirac field in the closed FRW universe. It is generally expected that the comoving energy density either a4 for relativistic or a3 for non-relativistic particles is conserved. Although this expectation is true for the massless fields, it is not for the massive ones. So, there is a finite energy current in any finite sized volume of the universe. In fig. $1 \mathrm{a}$, for $\mathrm{n}=0$, energy current is positive throughout. The maximum energy increase is near $r \approx \pi / 2$. Larger $l$ makes the peak sharper. For $n$ $=1$, however, there occurs negative energy flow near $r=\pi / 2$. So energy flows out of the region. It may be expected that the two regions, on either sides may become disjoint over time, but this can be confirmed only after studying the time evolution. If confirmed, this could explain the process of fragmentation of large structures. Larger $n$ appears to produce larger number of such density contrasts.

\section{REFERENCES}

[1] Newman, E.T. and Penrose, R. 1962. J. Math. Phys. 3: 566.

[2] Khanal, U. 2006. Class. Quantum Grav. 23: 4353.

[3] Chandrasekhar, S. 1983. The Mathematical Theory of Black Holes, Clarendon Press, Oxford).

[4] Gradshteyn, I.S. and Ihzhik, I.M. 2000. Table of Integrals, Series, and Products, Sixth Edition, Academic Press.

[5] Khanal, U. 2006. ICTP Preprint. IC/2006/136. 